\title{
On the number of limit cycles of polynomial Liénard systems*
}

\author{
Maoan Han ${ }^{a, b \dagger}$, Valery G. Romanovskib ${ }^{b, c \ddagger}$ \\ a Department of Mathematics, Shanghai Normal University, \\ Shanghai 200234, P. R. China \\ ${ }^{b}$ Faculty of Natural Science and Mathematics, \\ University of Maribor, SI-2000 Maribor, Slovenia \\ ${ }^{c}$ CAMTP - Center for Applied Mathematics and Theoretical Physics, \\ University of Maribor, SI-2000 Maribor, Slovenia
}

\begin{abstract}
Liénard systems are very important mathematical models describing oscillatory processes arising in applied sciences. In this paper, we study polynomial Liénard systems of arbitrary degree on the plane, and develop a new method to obtain a lower bound of the maximal number of limit cycles. Using the method and basing on some known results for lower degree we obtain new estimations of the number of limit cycles in the systems which greatly improve existing results.
\end{abstract}

Keywords: Limit cycle, polynomial Liénard system, global bifurcation.

\section{Introduction and main results}

Consider a polynomial Liénard system of the form

$$
\dot{x}=y, \quad \dot{y}=-g(x)-\varepsilon f(x) y,
$$

*The project supported by the National Natural Science Foundation of China (10971139) and the Slovenian Research Agency.

${ }^{\dagger}$ Corresponding author. E-mail address: mahan@shnu.edu.cn; tel. +8621-64328672; fax +862164328672

${ }^{\ddagger}$ E-mail address: valery.romanovsky@uni-mb.si. 
where $\varepsilon$ is a small parameter, $f(x), g(x)$ are polynomials in $x$ of degree $n$ and $m$, respectively. The above system is called a Liénard system. It describes the dynamics of systems of one degree of freedom under existence of a linear restoring force and a nonlinear dumping. It was shown by Liénard [1] that under some conditions on the functions $f(x)$ and $g(x)$ in the system arise auto-oscillations. In the first half of the last century models based on the Liénard system were important for the development of radio and vacuum tube technology. Nowadays the system is widely used to describe oscillatory processes arising in various studies of mathematical models of physical, biological, chemical, epidemiological, physiological, economical and many other phenomena (see e.g. [2, 3] and references therein).

Our study is devoted to finding Liénard systems which admit not a single, but few auto-oscillatory regimes (limit cycles). Let $H(n, m)$ denote the maximal number of limit cycles of system (1.1) on the plane for $\varepsilon$ sufficiently small. The lower bound of $H(n, m)$ for the Liénard system has been widely studied. For general $m$ and $n$ using the averaging theory of order 3 , the authors of [4] gave the estimation

$$
H(n, m) \geq\left[\frac{n+m-1}{2}\right]
$$

which generalized the earlier bound $H(n, 1) \geq\left[\frac{n}{2}\right]$ of Blows and Lloyd []. Recently, Han, Tian and $\mathrm{Yu}$ [6] obtained the following improvement

$$
H(n, m) \geq \max \left\{\left[\frac{m-2}{3}\right]+\left[\frac{2 n+1}{3}\right],\left[\frac{n-2}{3}\right]+\left[\frac{2 m+1}{3}\right]\right\}
$$

for $m, n \geq 2$. For $m=2$, Han [7] proved $H(n, 2) \geq\left[\frac{2 n+1}{3}\right], n \geq 2$. For $m=3$, Dumortier and $\mathrm{Li}[$ ] obtained $H(2,3) \geq 5$, Christopher and Lynch [9] proved

$$
H(n, 3) \geq 2\left[\frac{3 n+6}{8}\right], 2 \leq n \leq 50
$$

and Yang, Han and Romanovski [10] further obtained

$$
H(n, 3) \geq\left[\frac{3 n+14}{4}\right], 2 \leq n \leq 8,
$$

which gave a larger lower bound of $H(n, 3)$ than the above ones for $3 \leq n \leq 8$.

From [11, 12] we know that

$$
H(n, 3) \geq n+2-\left[\frac{n+1}{4}\right], \quad 9 \leq n \leq 22 .
$$

For $m=4$, Han, Yan, Yang and Lhotka [13] studied the limit cycle bifurcation of system (1.1) and obtained

$$
H(n, 4) \geq n+3, n=2,3,5,6,7,8, H(4,4) \geq 6 .
$$


Christopher and Lynch [9] gave $H(9,4) \geq 9$. And then, Yu and Han [14, 15] obtained

$$
\begin{gathered}
H(n, 4) \geq n, n=10,11,12,13,14 . \\
H(n, 4) \geq n+4-\left[\frac{n+1}{5}\right], 3 \leq n \leq 18 .
\end{gathered}
$$

More results for some concrete $m$ and $n$ can be found in [14].

Motivated by [16], in this paper we give a new method to find a lower bound of $H(n, m)$ for many integers $m$ and $n$. The main results are the following.

(1) $H(n, 4) \geq H(n, 3) \geq 2\left[\frac{n-1}{4}\right]+\left[\frac{n-1}{2}\right], n \geq 3$.

(2) $H(n, 6) \geq H(n, 5) \geq 2\left[\frac{n-1}{3}\right]+\left[\frac{n-1}{2}\right], n \geq 5$.

(3) $H(n, 7) \geq \frac{3}{2} n-9$ for $n \geq 7$. In general, for any integer $m \geq 7$ there exists $\gamma_{m}>0$ satisfying

$$
\lim _{m \rightarrow \infty} \sup \frac{\gamma_{m}}{(m+1) \ln (m+1)} \leq \frac{1}{4 \ln 2}
$$

such that

$$
H(n, m) \geq\left(\frac{\ln (m+2)}{2 \ln 2}-\frac{1}{3}\right) n-\gamma_{m}, \quad n \geq m .
$$

In particular,

$$
\lim _{m \rightarrow \infty} \lim _{n \rightarrow \infty} \inf \frac{H(n, m)}{n \ln (m+2)} \geq \frac{1}{2 \ln 2} .
$$

(4) For any integer $r \geq 0$,

$$
\lim _{m \rightarrow \infty} \inf \frac{H(m \pm r, m)}{m \ln m} \geq \frac{1}{2 \ln 2} .
$$

(5) For $m=2^{p+1}-1, p \geq 1$, we have

$$
H(m-1, m) \geq \frac{(m+1) \ln (m+1)}{2 \ln 2}+1,
$$

and

$$
H(m, m) \geq \frac{(m+1) \ln (m+1)}{2 \ln 2}+1 .
$$

(6) For all $m \geq 3$ we have

$$
H(m-1, m) \geq \frac{(m+2) \ln (m+2)}{3 \ln 2}-\frac{m+2}{3}\left(1+\frac{\ln 3}{\ln 2}\right)+1,
$$

and

$$
H(m, m) \geq \frac{(m+2) \ln (m+2)}{3 \ln 2}-\frac{m+2}{3}\left(1+\frac{\ln 3}{\ln 2}\right)+1 .
$$

(7) Let $r$ be a positive integer. For any $k \geq 2$ there exist constants $B_{k, r}$ and $\bar{B}_{k, r}$ satisfying

$$
B_{k, r} \geq-\left[\frac{1}{k}\left(1+\left[\frac{r}{2}\right]\right)+\frac{\ln k}{2 \ln 2}\right]
$$




$$
\bar{B}_{k, r} \geq-\left[\frac{1}{k+1}\left(2+\left[\frac{r}{2}\right]\right)+\frac{\ln (k+1)}{2 \ln 2}\right]
$$

such that

$$
H(m-r, m) \geq \frac{(m+1) \ln (m+1)}{2 \ln 2}+B_{k, r}(m+1)+1+\left[\frac{r}{2}\right]
$$

for $m=2^{p} k-1, p \geq 1$, and

$$
H(m-r, m) \geq \frac{(m+2) \ln (m+2)}{2 \ln 2}+\bar{B}_{k, r}(m+2)+2+\left[\frac{r}{2}\right]
$$

for $m=2^{p}(k+1)-2, p \geq 1$.

The conclusions listed above are contained in Theorems 3.1, 3.2, 4.2, 4.3, 5.1, 5.2 and formula (5.1) in sections 3,4 and 5 below.

\section{Preliminary results}

The polynomial Liénard system (1.1) can be transformed to the form

$$
\dot{x}=y-\varepsilon F(x), \dot{y}=-g(x),
$$

where $F(x)=\int_{0}^{x} f(x) d x$.

We introduce the following definition.

Definition 2.1. We say that the system (2.1) has property $Z(n, m, k)$ if the following are satisfied:

(1) $\operatorname{deg} F \leq n+1, \operatorname{deg} g \leq m$, and $\lim _{x \rightarrow+\infty} g(x)=+\infty$;

(2) there are a constant $\varepsilon_{0}>0$ and a compact set $D \subset \mathbb{R}^{2}$ such that for all $0<\varepsilon \leq \varepsilon_{0}$ the system (2.1) has at least $k$ limit cycles in $D$, each having an odd multiplicity.

Obviously, if we can find a polynomial system (2.1) which has the property $Z(n, m, k)$, then $H(n, m) \geq k$.

As we know, the first order Melnikov function of (2.1) has the form

$$
M(h)=\oint_{L_{h}} F(x) d y,
$$

where $L_{h}$ is a smooth closed curve defined by the equation $H(x, y)=h$ on the plane, with

$$
H(x, y)=\frac{1}{2} y^{2}+G(x), \quad G(x)=\int_{0}^{x} g(x) d x .
$$

As it is known [17, (2.1) has the property $Z(n, m, k)$ if the function $M(h)$ has at least $k$ zeros, each having an odd multiplicity.

From the work of [5, 7, 10, 15], we know that the following facts hold.

Lemma 2.1. There are polynomial Liénard systems of the form (2.1) which have the following properties 
(1) $Z\left(n, 1,\left[\frac{n}{2}\right]\right)$ and $Z\left(n, 2,\left[\frac{2 n+1}{3}\right]\right)$ for $n \geq 1$;

(2) $Z\left(n, 3,\left[\frac{3 n+14}{4}\right]\right)$ for $2 \leq n \leq 8$ and $Z\left(n, 4, n+4-\left[\frac{n+1}{5}\right]\right)$ for $3 \leq n \leq 18$.

Next we give a method to construct polynomial Liénard systems having new properties starting from a given polynomial Liénard system having a certain property. We begin with the following lemma.

Lemma 2.2. Let (2.1) have the property $Z(n, m, k)$. Then there exists $x^{*}>0$ such that for all $x_{0}<-x^{*}$ the system

$$
\dot{x}=y-\varepsilon F\left(x^{2}+x_{0}\right), \dot{y}=-2 x g\left(x^{2}+x_{0}\right)
$$

has the property $Z(2 n+1,2 m+1,2 k)$.

Proof. By Definition 2.1, there are a constant $\varepsilon_{0}>0$ and a compact set $D \subset \mathbb{R}^{2}$ such that for all $0<\varepsilon \leq \varepsilon_{0}$ the system (2.1) has at least $k$ limit cycles in $D$, each having an odd multiplicity. Since $D$ is compact, for some $x^{*}>0$ we must have $D \subset\left\{(x, y)|| x \mid<x^{*}\right\}$. Let $x_{0}<-x^{*}$. Then for $|x|<x^{*}$ we have $x-x_{0}>0$. Note that the change $u=x-x_{0}$ carries (2.1) into

$$
\dot{u}=y-\varepsilon F\left(u+x_{0}\right), \dot{y}=-g\left(u+x_{0}\right) .
$$

Thus, the above system has $k$ limit cycles on a compact set which is contained in $\{(u, y) \mid u>$ $0\}$.

Further, we introduce $u=v^{2}$ to (2.3) to obtain

$$
2 v \dot{v}=y-\varepsilon F\left(v^{2}+x_{0}\right), \quad 2 v \dot{y}=-2 v g\left(v^{2}+x_{0}\right)
$$

which is equivalent to

$$
\dot{v}=y-\varepsilon F\left(v^{2}+x_{0}\right), \dot{y}=-2 v g\left(v^{2}+x_{0}\right)
$$

on $v<0$ or $v>0$. Therefore, (2.4) has $k$ limit cycles both on a compact set $D_{1}$ in $v<0$ and on a compact set $D_{2}$ in $v>0$. That is to say, it has $2 k$ limit cycles on $D_{1} \cup D_{2}$. The conclusion follows. This ends the proof.

Lemma 2.3. Suppose $G_{1}(x)$ is a polynomial of degree $2 l$ satisfying

$$
G_{1}(x)=G_{0} x^{2 l}\left(1+O\left(x^{-1}\right)\right), l \geq 1, G_{0}>0
$$

as $|x| \rightarrow \infty$. Let $H_{1}(x, y)=\frac{1}{2} y^{2}+G_{1}(x)$ and

$$
I_{j}(h)=\oint_{H_{1}=h} x^{2 j+1} d y, \quad j \geq 0
$$

along the orbits of the system

$$
\dot{x}=y, \quad \dot{y}=-G_{1}^{\prime}(x) .
$$


Then there exist constants $\alpha_{j}>0, j \geq 0$ such that

$$
I_{j}(h)=-\alpha_{j} h^{\frac{2 j+l+1}{2 l}}(1+o(1))
$$

as $h \rightarrow \infty$.

Proof. Noting $y d y=-G_{1}^{\prime}(x) d x$ along $H_{1}(x, y)=h$, we have

$$
I_{j}(h)=-\oint_{H_{1}=h} \frac{x^{2 j+1} G_{1}^{\prime}(x)}{y} d x=-2 \int_{a(h)}^{b(h)} \frac{x^{2 j+1} G_{1}^{\prime}(x)}{\sqrt{2\left(h-G_{1}(x)\right)}} d x,
$$

where $a(h)$ and $b(h)$ are the solutions of the equation $G_{1}(x)=h$ satisfying

$$
\lim _{h \rightarrow \infty} a(h)=-\infty, \lim _{h \rightarrow \infty} b(h)=\infty .
$$

Let $x_{0}>0$ and $x_{0}^{\prime}<0$ be such that

$$
G_{1}\left(x_{0}\right)=G_{1}\left(x_{0}^{\prime}\right) \equiv u_{0}>0, G_{1}(x)>0, x G_{1}^{\prime}(x)>0 \text { for } x_{0}^{\prime} \leq x \leq x_{0} .
$$

Then

$$
I_{j}(h)=\tilde{I}_{1}(h)+\tilde{I}_{2}(h)+\tilde{I}_{3}(h),
$$

where

$$
\begin{gathered}
\tilde{I}_{1}(h)=-2 \int_{a(h)}^{x_{0}^{\prime}} \frac{x^{2 j+1} G_{1}^{\prime}(x)}{\sqrt{2\left(h-G_{1}(x)\right)}} d x=2 \int_{u_{0}}^{h} \frac{\left(x_{1}(h)\right)^{2 j+1}}{\sqrt{2(h-u)}} d u, \\
\tilde{I}_{2}(h)=-2 \int_{\left.x_{0}\right)}^{b(h)} \frac{x^{2 j+1} G_{1}^{\prime}(x)}{\sqrt{2\left(h-G_{1}(x)\right)}} d x=-2 \int_{u_{0}}^{h} \frac{\left(x_{2}(h)\right)^{2 j+1}}{\sqrt{2(h-u)}} d u, \\
\tilde{I}_{3}(h)=-2 \int_{x_{0}^{\prime}}^{x_{0}} \frac{x^{2 j+1} G_{1}^{\prime}(x)}{\sqrt{2\left(h-G_{1}(x)\right)}} d x,
\end{gathered}
$$

and $x_{1}(u)<0<x_{2}(u)$ satisfy $G_{1}\left(x_{i}(u)\right)=u$ for $u_{0} \leq u \leq h, i=1,2$. Obviously, $\lim _{h \rightarrow \infty} \tilde{I}_{3}(h)=0$. Thus, to finish the proof we need only to prove that there exist constants $\beta_{1}>0$ and $\beta_{2}>0$ such that

$$
\tilde{I}_{i}(h)=-\beta_{i} h^{\frac{2 j+l+1}{2 l}}(1+o(1)), i=1,2
$$

as $h \rightarrow \infty$. We only consider the case $i=1$. The case $i=2$ is just similar. By our assumption on $G_{1}$, the equation $G_{1}(x)=u$ can be rewritten as

$$
|x|\left(1+O\left(|x|^{-1}\right)\right)=\left(u / G_{0}\right)^{\frac{1}{2 l}}
$$

for $u>0$ large. It then follows that

$$
x_{1}(u)=-\left(u / G_{0}\right)^{\frac{1}{2 l}}\left(1+O\left(u^{\frac{-1}{2 l}}\right)\right) .
$$

Hence

$$
\begin{aligned}
\left(x_{1}(h)\right)^{2 j+1} & =-\left(u / G_{0}\right)^{\frac{2 j+1}{2 l}}\left(1+O\left(u^{\frac{-1}{2 l}}\right)\right) \\
& =-\left(u / G_{0}\right)^{\frac{2 j+1}{2 l}}+u^{\frac{j}{l}} \varphi_{1}(u),
\end{aligned}
$$


where $\varphi_{1}(u)$ is smooth and bounded on the interval $\left[u_{0},+\infty\right)$.

Now introducing the change $u=h \sin ^{2} \theta$ we obtain from (2.5)

$$
\begin{aligned}
\left.\tilde{I}_{1}(h)\right) & =-2 \int_{\theta_{0}(h)}^{\frac{\pi}{2}} \sqrt{2 h} \sin \theta\left(x_{1}\left(h \sin ^{2} \theta\right)\right)^{2 j+1} d \theta \\
& =-h^{\frac{2 j+1}{2 l}+\frac{1}{2}} \tilde{\varphi}_{0}(h)+h^{\frac{j}{l}+\frac{1}{2}} \tilde{\varphi}_{1}(h),
\end{aligned}
$$

where

$$
\tilde{\varphi}_{0}(h)=2 \sqrt{2} G_{0}^{-\frac{2 j+1}{2 l}} \int_{\theta_{0}(h)}^{\frac{\pi}{2}}[\sin \theta]^{\frac{2 j+1}{l}+1} d \theta,
$$

$\tilde{\varphi}_{1}(h)$ is bounded on $\left[u_{0},+\infty\right)$, and $\theta_{0}(h)$ satisfies $\sin \theta_{0}=\sqrt{u_{0} / h}$. It is evident that

$$
\lim _{h \rightarrow \infty} \tilde{\varphi}_{0}(h)=2 \sqrt{2} G_{0}^{-\frac{2 j+1}{2 l}} \int_{0}^{\frac{\pi}{2}}[\sin \theta]^{\frac{2 j+1}{l}+1} d \theta \equiv \beta_{1} .
$$

Then (2.6) follows for $i=1$. This completes the proof.

Using the above lemma, we have further the following fundamental lemma.

Lemma 2.4. Let $G_{2}(x)$ and $F_{2}(x)$ be even polynomials in $x$ with $G_{2}(\infty)=+\infty$. Then for any integer $q \geq 1$ and a compact set $U_{0} \subset \mathbb{R}^{2}$ there exist $\varepsilon_{0}>0, b_{j} \neq 0$, $j=0, \cdots, q$ and a compact set $U \subset \mathbb{R}^{2}$ with $U \cap U_{0}=\emptyset$ such that the following system

$$
\begin{aligned}
& \dot{x}=y-\left[\lambda F_{2}(x)+\mu \sum_{j=0}^{q} b_{j} x^{2 j+1}\right], \\
& \dot{y}=-G_{2}^{\prime}(x)
\end{aligned}
$$

has $q$ limit cycles with odd multiplicity in $U$ for all $|\lambda| \leq \varepsilon_{0}$ and $0<|\mu| \leq \varepsilon_{0}$.

Proof. Since $G_{2}$ is even with $G_{2}(\infty)=+\infty$, there exists $x_{0}>0$ such that for all $a \geq x_{0}$ the orbit $\gamma(a)$ of the Hamiltonian system $\dot{x}=y, \dot{y}=-G_{2}^{\prime}(x)$ starting from $A(a, 0)$ is periodic, which ensures $G_{2}^{\prime}(x)>0$ for $a>x_{0}$. Also, for any given compact set $U_{0} \subset \mathbb{R}^{2}$ there is $h^{*}>G_{2}\left(x_{0}\right) \equiv h_{0}$ such that $U_{0} \subset\left\{(x, y) \mid H_{2}(x, y)<h^{*}\right\}$.

Hence, for any given $x_{1}>x_{0}$ satisfying $h^{*}<G_{2}\left(x_{1}\right) \equiv h_{1}$ there exists $\varepsilon_{1}=\varepsilon_{1}\left(x_{1}\right)>0$ such that for all $a \in\left[x_{0}, x_{1}\right]$ and $|\lambda| \leq \varepsilon_{1}$ the orbit $\gamma_{\lambda}(a)$ of the symmetric system

$$
\dot{x}=y-\lambda F_{2}(x), \quad \dot{y}=-G_{2}^{\prime}(x)
$$

starting from the same point $A(a, 0)$ is also periodic. Then further, for any given $N>0$ there exists $\varepsilon_{2}=\varepsilon_{2}\left(x_{1}, N\right)>0$ such that for all $a \in\left[x_{0}, x_{1}\right],|\lambda| \leq \varepsilon_{1},|\mu| \leq \varepsilon_{2}$ and $\left|b_{j}\right| \leq N, j=0, \ldots, q$ the system (2.7) has a positive orbit $\gamma_{\lambda, \mu}^{+}(a)$ starting from the point $A(a, 0)$ which intersects the positive $x$-axis again at some point $B(b(a, \lambda, \mu), 0)$ for the first time. To find $\varepsilon_{0}$ and $U$, suitable $x_{1}$ and $N$ will be chosen later. Let

$$
H_{2}(x, y)=\frac{1}{2} y^{2}+G_{2}(x)
$$


Then along the orbit $\widehat{A B}$ of (2.7) we have

$$
\begin{aligned}
H_{2}(B)-H_{2}(A) & =\int_{\widehat{A B}}\left(G_{2}^{\prime}(x) d x+y d y\right) \\
& =\int_{\widehat{A B}}\left(\lambda F_{2}(x)+\mu \sum_{j=0}^{q} b_{j} x^{2 j+1}\right) G_{2}^{\prime}(x) d t .
\end{aligned}
$$

Note that $b(a, \lambda, 0)=a$ for all $a \in\left[x_{0}, x_{1}\right]$ and $|\lambda| \leq \varepsilon_{1}$. We have $H_{2}(B)-H_{2}(A)=$ $O(\mu)$ which gives

$$
\begin{aligned}
-\int_{\widehat{A B}} F_{2}(x) G_{2}^{\prime}(x) d t=\mu \varphi(a, \lambda, \mu) & \\
\int_{\widehat{A B}} x^{2 j+1} G_{2}^{\prime}(x) d t & =\oint_{\gamma_{\lambda}(a)} x^{2 j+1} G_{2}^{\prime}(x) d t+\mu \psi_{j}(a, \lambda, \mu) \\
& =-\oint_{\gamma(a)} x^{2 j+1} d y+\lambda \varphi_{j}(a, \lambda, \mu)+\mu \psi_{j}(a, \lambda, \mu)
\end{aligned}
$$

where $\varphi, \varphi_{j}$ and $\psi_{j}$ are smooth functions for $a \in\left[x_{0}, x_{1}\right],|\lambda| \leq \varepsilon_{1}$ and $|\mu| \leq \varepsilon_{2}$. Thus, we have

$$
H_{2}(B)-H_{2}(A)=\mu\left[M(h)+\lambda \varphi+\sum_{j=0}^{q} b_{j}\left(\lambda \varphi_{j}+\mu \psi_{j}\right)\right] \equiv \mu d(h, \lambda, \mu),
$$

where

$$
M(h)=\sum_{j=0}^{q} b_{j} I_{j}(h), \quad I_{j}(h)=\oint_{H_{2}=h} x^{2 j+1} d y, h=G_{2}(a) \in\left[h_{0}, h_{1}\right] .
$$

Let $\operatorname{deg} G_{2}(x)=2 l$. Then by Lemma 2.3 , we see that

$$
\frac{I_{j+1}}{I_{j}}=\frac{\alpha_{j+1}}{\alpha_{j}} h^{\frac{1}{l}}(1+o(1)), j=0,1, \cdots, q-1
$$

for $h \gg 1$. Hence, we first fix $b_{0} \neq 0$ and then vary $b_{1}, b_{2}, \cdots, b_{q}$ in turn satisfying

$$
\left|b_{q}\right| \ll\left|b_{q-1}\right| \ll \cdots \ll\left|b_{0}\right|, b_{j} b_{j+1}<0, j=0, \cdots, q-1
$$

such that $M(h)$ has $q$ zeros, denoted by $\bar{h}_{i}, i=1, \cdots, q$, on the interval $\left[h^{*},+\infty\right)$, each having an odd multiplicity.

Now we fix $b_{j}$ as taken before, and then choose $N, h_{1}, x_{1}$ and $\bar{\varepsilon}_{0}$ as follows:

$$
\begin{aligned}
& N=\max _{0 \leq j \leq q}\left\{\left|b_{j}\right|\right\}, \\
& h_{1}=\max _{1 \leq i \leq q}\left\{\bar{h}_{i}\right\}+1=G_{2}\left(x_{1}\right), \\
& \bar{\varepsilon}_{0}=\max \left\{\varepsilon_{1}\left(x_{1}\right), \varepsilon_{2}\left(x_{1}, N\right)\right\} .
\end{aligned}
$$

Then, by the above discussion, the function $d(h, \lambda, \mu)$ in (2.8) is well defined for all $h \in\left[h_{0}, h_{1}\right],|\lambda| \leq \bar{\varepsilon}_{0},|\mu| \leq \bar{\varepsilon}_{0}$, and the function $M(h)$ has $q$ different zeros with odd multiplicity on the open interval $\left(h^{*}, h_{1}\right)$. Let

$$
U=\left\{(x, y) \mid h^{*} \leq H(x, y) \leq h_{1}\right\}
$$


Since all of the $q$ zeros of $M$ have an odd multiplicity, there exists an $\varepsilon_{0} \in\left(0, \bar{\varepsilon}_{0}\right)$ such that for all $|\lambda| \leq \varepsilon_{0},|\mu| \leq \varepsilon_{0}$ the function $d(h, \lambda, \mu)$ has $q$ zeros in $h \in\left(h^{*}, h_{1}\right)$, each having an odd multiplicity, and that the corresponding limit cycles of (2.7) are all located in $U$. This ends the proof.

The main result of this section is the following theorem.

Theorem 2.1. If (2.2) has the property $Z(n, m, k)$, then there exist two polynomial systems of the form

$$
\dot{x}=y-\varepsilon F_{2 n+2}, \quad \dot{y}=-g_{2 m+1}(x),
$$

and

$$
\dot{x}=y-\varepsilon F_{2 n+3}, \quad \dot{y}=-g_{2 m+1}(x),
$$

which have properties $Z(2 n+1,2 m+1,2 k+n)$ and $Z(2 n+2,2 m+1,2 k+n+1)$, respectively, where

$$
\begin{aligned}
& 2 n+1 \leq \operatorname{deg} F_{2 n+2} \leq 2 n+2, \quad \operatorname{deg} F_{2 n+3}=2 n+3, \\
& 3 \leq \operatorname{deg} g_{2 m+1} \leq 2 m+1, \quad g_{2 m+1}(-x)=-g_{2 m+1}(x) .
\end{aligned}
$$

Proof. First, by Lemma 2.2, there are a constant $\varepsilon_{0}>0$ and a compact set $U_{0} \subset \mathbb{R}^{2}$ such that for all $0<\varepsilon \leq \varepsilon_{0}$ the system (2.2) has at least $2 k$ limit cycles in $U_{0}$, each having an odd multiplicity. Let

$$
F_{2}(x)=F\left(x^{2}+x_{0}\right), \quad G_{2}(x)=\int_{0}^{x} 2 x g\left(x^{2}+x_{0}\right) d x .
$$

Then $\operatorname{deg} F_{2}(x) \leq 2 n+2, \operatorname{deg} G_{2}(x)=2 l, 2 \leq l \leq m+1$. Consider (2.7) with $q=n$ or $n+1$. For each fixed $\lambda$ with $0<|\lambda| \leq \varepsilon_{0}$, (2.7) has $2 k$ limit cycles in $U_{0}$ for all $0<|\mu| \ll|\lambda|$, each having an odd multiplicity. On the other hand, by Lemma 2.4, there exist $\varepsilon^{*} \in\left(0, \varepsilon_{0}\right)$, constants $b_{j} \neq 0, j=0,1, \cdots, q$ and a compact set $U$ with $U \cap U_{0}=\emptyset$ such that for all $|\lambda| \leq \varepsilon^{*}, 0<|\mu| \leq \varepsilon^{*}$, (2.7) has $q$ limit cycles in $U$, each having an odd multiplicity. Therefore, for all $(\lambda, \mu)$ satisfying

$$
0<|\mu| \ll|\lambda| \leq \varepsilon^{*}
$$

(2.7) has $2 k+q$ limit cycles in the set $U \bigcup U_{0}$. Then set $g_{2 m+1}(x)=G_{2}^{\prime}(x)$, and

$$
F_{2 n+2}(x)=\lambda F_{2}(x)+\mu \sum_{j=0}^{n} b_{j} x^{2 j+1}, F_{2 n+3}(x)=\lambda F_{2}(x)+\mu \sum_{j=0}^{n+1} b_{j} x^{2 j+1} .
$$

It follows that for all $0<\varepsilon \leq 1$ and some $(\lambda, \mu)$ satisfying (2.11), the system (2.9) has the property $Z(2 n+1,2 m+1,2 k+n)$, and (2.10) has property $Z(2 n+2,2 m+1,2 k+n+1)$. This finishes the proof.

An obvious corollary of the theorem above is the following. 
Corollary 2.1. If (2.2) has the property $Z(n, m, k)$, then there exist two polynomial systems of the form

$$
\dot{x}=y-\varepsilon F_{2 n+2}, \quad \dot{y}=-g_{2 m+2}(x)
$$

and

$$
\dot{x}=y-\varepsilon F_{2 n+3}, \quad \dot{y}=-g_{2 m+2}(x)
$$

which has properties $Z(2 n+1,2 m+2,2 k+n)$ and $Z(2 n+2,2 m+2,2 k+n+1)$, respectively.

The theorem above together with Corollary 2.1 is fundamental. We can use them repeatedly. See the next two sections.

\section{Estimate of $H(n, m)$ for fixed $m$}

Suppose there is a system of the form (2.1) which has property $Z\left(n_{0}, m_{0}, k_{0}\right)$ with $n_{0} \geq 1$, $m_{0} \geq 1$. Then define

$$
\begin{gathered}
n_{11}=2 n_{0}+1, n_{12}=2 n_{0}+2, \\
m_{11}=2 m_{0}+1, m_{12}=2 m_{0}+2, \\
k_{11}=2 k_{0}+n_{0}, k_{12}=2 k_{0}+n_{0}+1 .
\end{gathered}
$$

Then by Theorem 2.1 and Corollary 2.1, there are polynomial Liénard systems of the form (2.1) which have 4 properties $Z\left(n_{1 i}, m_{1 j}, k_{1 i}\right)$ for $i, j=1,2$, respectively, which imply

$$
H\left(n_{1 i}, m_{1 j}\right) \geq k_{1 i}, i, j=1,2 .
$$

Hence, using Lemma 2.1 and formulas in (3.1) and (3.2) we obtain the following.

Theorem 3.1. We have

(1) $H(n, 4) \geq H(n, 3) \geq 2\left[\frac{n-1}{4}\right]+\left[\frac{n-1}{2}\right], n \geq 3$;

(2) $H(n, 6) \geq H(n, 5) \geq 2\left[\frac{n-1}{3}\right]+\left[\frac{n-1}{2}\right], n \geq 3$.

Proof. We only prove the first conclusion. The second one can be shown similarly. For any integer $\tilde{n} \geq 3$, let $n=\left[\frac{\tilde{n}-1}{2}\right]$. Then $n \geq 1$, and either $\tilde{n}=2 n+1$ or $\tilde{n}=2 n+2$. By Lemma 2.1, we have property $Z\left(n, 1,\left[\frac{n}{2}\right]\right)$. Then by (3.1) we further obtain properties $Z\left(2 n+1, j, 2\left[\frac{n}{2}\right]+n\right)$ and $Z\left(2 n+2, j, 2\left[\frac{n}{2}\right]+n+1\right), j=3,4$. Using (3.2) we conclude

$$
H(2 n+1, j) \geq 2\left[\frac{n}{2}\right]+n, H(2 n+2, j) \geq 2\left[\frac{n}{2}\right]+n+1, j=3,4 .
$$

It follows that

$$
H(\tilde{n}, j) \geq 2\left[\frac{1}{2}\left[\frac{\tilde{n}-1}{2}\right]\right]+\left[\frac{\tilde{n}-1}{2}\right]=2\left[\frac{\tilde{n}-1}{4}\right]+\left[\frac{\tilde{n}-1}{2}\right] .
$$

This finishes the proof. 
By Theorem 2.1 and Corollary 2.1, we can get more results. For the purpose, let

$$
S_{m_{0}}=\left\{2^{i}\left(m_{0}+1\right)-2+j \mid 1 \leq j \leq 2^{i}, i \geq 0\right\}
$$

for $m_{0} \geq 1$, It is easy to see that $S_{1} \bigcup S_{2}=\{m \mid m \geq 1\}$. Thus by Lemma 3.2 in [16], for any integer $M \geq 1$,

$$
\bigcup_{m_{0}=M}^{2 M} S_{m_{0}}=\{m \mid m \geq M\} .
$$

Theorem 3.2. We have

(1) For any integers $p \geq 1$ and $1 \leq j \leq 2^{p}$ there exist constants $0<\delta_{p}<(p+4) 2^{p-1}-$ $(p+1)$ and $0<\beta_{p}<\left(p+\frac{13}{3}\right) 2^{p-1}-\left(p+\frac{4}{3}\right)$ such that

$$
\begin{aligned}
& H\left(n, 2^{p+1}-2+j\right) \geq \frac{1}{2}(p+1) n-\delta_{p}, \\
& H\left(n, 3 \cdot 2^{p}-2+j\right) \geq\left(\frac{1}{2} p+\frac{2}{3}\right) n-\beta_{p} .
\end{aligned}
$$

In particular,

$$
H(n, 7) \geq \frac{3}{2} n-9 \text { for } n \geq 7 .
$$

(2) For any integer $m \geq 7$ there exists $\gamma_{m}>0$ satisfying

$$
\lim _{m \rightarrow \infty} \sup \frac{\gamma_{m}}{(m+1) \ln (m+1)} \leq \frac{1}{4 \ln 2}
$$

such that

$$
H(n, m) \geq\left(\frac{\ln (m+2)}{2 \ln 2}-\frac{1}{3}\right) n-\gamma_{m}, \quad n \geq m .
$$

In particular,

$$
\lim _{m \rightarrow \infty} \lim _{n \rightarrow \infty} \inf \frac{H(n, m)}{n \ln (m+2)} \geq \frac{1}{2 \ln 2} .
$$

Proof. Note that $\left[\frac{n}{2}\right] \geq \frac{n}{2}-\frac{1}{2}$ for all $n \geq 1$. We have

$$
2\left[\frac{n}{2}\right]+n \geq 2\left(\frac{n}{2}-\frac{1}{2}\right)+n=2 n-1,2\left[\frac{n-1}{2}\right]-1 \geq 2\left(\frac{n-1}{2}-\frac{1}{2}\right)-1=n-3 .
$$

Hence, the first conclusion of Theorem 3.1 implies

$$
H\left(n, 2^{2}-2+j\right) \geq l_{1} n-\delta_{1}, n \geq 3, j=1,2,
$$

where $l_{1}=1, \delta_{1}=3$. Just following the idea in the proof of Theorem 3.1, we can obtain further

$$
H\left(2 n+2,2^{3}-1\right) \geq H\left(2 n+1,2^{3}-1\right) \geq 2\left(l_{1} n-\delta_{1}\right)+n, \quad n \geq 2^{2}-1,
$$

which implies

$$
\begin{aligned}
H\left(n, 2^{3}-1\right) & \geq\left(2 l_{1}+1\right)\left[\frac{n-1}{2}\right]-2 \delta_{1} \\
& \geq\left(2 l_{1}+1\right)\left(\frac{n-1}{2}-\frac{1}{2}\right)-2 \delta_{1} \\
& =l_{2} n-\delta_{2}, n \geq 2^{3}-1,
\end{aligned}
$$


where $l_{2}=l_{1}+\frac{1}{2}=\frac{3}{2}, \delta_{2}=9$. In particular, (3.6) follows. Note that $H\left(n, 2^{3}-2+j\right) \geq$ $H\left(n, 2^{3}-1\right)$ for $1 \leq j \leq 2^{2}$. Hence, we have

$$
H\left(n, 2^{3}-2+j\right) \geq l_{2} n-\delta_{2}, n \geq 2^{3}-1,1 \leq j \leq 2^{2}
$$

In the same way, we have for $p \geq 3$

$$
H\left(n, 2^{p+1}-2+j\right) \geq l_{p} n-\delta_{p}, n \geq 2^{p+1}-1,1 \leq j \leq 2^{p},
$$

where $l_{p}$ and $\delta_{p}$ satisfy

$$
l_{p}=l_{p-1}+\frac{1}{2}, \quad \delta_{p}=2 \delta_{p-1}+2 l_{p-1}+1
$$

Then using these relations and the initial data $l_{1}=1$ and $\delta_{1}=3$, we can easily find

$$
l_{p}=\frac{p+1}{2}, \quad \delta_{p}=3 \cdot 2^{p-1}+\sum_{j=2}^{p}(j+1) 2^{p-j}, \quad p \geq 1 .
$$

Then noting

$$
\delta_{p} \leq 3 \cdot 2^{p-1}+(p+1) \sum_{j=2}^{p} 2^{p-j}=(p+4) 2^{p-1}-(p+1),
$$

(3.4) follows from (3.8).

Further, using $\left[\frac{n-1}{3}\right] \geq \frac{n-1}{3}-\frac{2}{3}$ it follows from the second conclusion of Theorem 3.1

$$
H(n, 6) \geq H(n, 5) \geq 2\left(\frac{n-1}{3}-\frac{2}{3}\right)+\frac{n-1}{2}-\frac{1}{2}=r_{1} n-\beta_{1},
$$

where $n \geq 5, r_{1}=\frac{7}{6}, \beta_{1}=3$.

Similar to the above, we can obtain for $p \geq 1$

$$
H\left(n, 3 \cdot 2^{p}-2+j\right) \geq r_{p} n-\beta_{p}, n \geq 3 \cdot 2^{p}-1,1 \leq j \leq 2^{p},
$$

where $r_{p}$ and $\beta_{p}$ satisfy

$$
r_{p}=r_{p-1}+\frac{1}{2}, \quad \beta_{p}=2 \beta_{p-1}+2 r_{p-1}+1
$$

which together with $r_{1}=\frac{7}{6}, \beta_{1}=3$ give

$$
r_{p}=\frac{p}{2}+\frac{2}{3}, \quad \beta_{p}=3 \cdot 2^{p-1}+\sum_{j=2}^{p}\left(j+\frac{4}{3}\right) 2^{p-j}, \quad p \geq 1 .
$$

Then noting

$$
\beta_{p} \leq 3 \cdot 2^{p-1}+\left(p+\frac{4}{3}\right) \sum_{j=2}^{p} 2^{p-j}=\left(p+\frac{13}{3}\right) 2^{p-1}-\left(p+\frac{4}{3}\right),
$$

(3.5) follows. 
By (3.3), any positive integer $m$ is either in $S_{1}$ or in $S_{2}$. If it is in $S_{1}$, then by the definition of $S_{m_{0}}$ there exist $p \geq 1$ and $1 \leq j \leq 2^{p}$ such that $m=2^{p+1}-2+j$, which implies $2^{p+1}-1 \leq m \leq 2^{p+1}-2+2^{p}=3 \cdot 2^{p}-2$, or $p+1 \leq \frac{1}{\ln 2} \ln (m+1), p \geq \frac{1}{\ln 2} \ln \frac{m+2}{3}$. Hence, by (3.8) and (3.9) we have

$$
H(n, m) \geq \bar{l}_{m} n-\bar{\delta}_{m}, \quad n \geq m
$$

where

$$
\bar{l}_{m}=\frac{1}{2}\left(1+\frac{1}{\ln 2} \ln \frac{m+2}{3}\right), \quad \lim _{m \rightarrow \infty} \sup \frac{\bar{\delta}_{m}}{(m+1) \ln (m+1)} \leq \frac{1}{4 \ln 2} .
$$

If $m \in S_{2}$, then similarly we have $m=3 \cdot 2^{p}-2+j$ for some $p \geq 0$ and $1 \leq j \leq 2^{p}$. Thus, $\frac{1}{\ln 2} \ln \frac{m+1}{3} \geq p \geq \frac{\ln (m+2)}{\ln 2}-2$. By (3.10) we have

$$
H(n, m) \geq \bar{r}_{m} n-\bar{\beta}_{m}, \quad n \geq m,
$$

where

$$
\bar{r}_{m}=\frac{\ln (m+2)}{2 \ln 2}-\frac{1}{3}, \quad \lim _{m \rightarrow \infty} \sup \frac{\bar{\beta}_{m}}{(m+1) \ln (m+1)} \leq \frac{1}{6 \ln 2} .
$$

Therefore, for $n \geq m$

$$
H(n, m) \geq \min \left\{\bar{l}_{m}, \bar{r}_{m}\right\} n-\max \left\{\bar{\delta}_{m}, \bar{\beta}_{m}\right\}
$$

which yields (3.7) since $\bar{r}_{m}<\bar{l}_{m}$. The proof is completed.

In (3.4) and (3.5), taking $j=1$ we have in particular

$$
H(n, m) \geq \frac{\ln (m+1)}{2 \ln 2} n-\bar{\delta}_{m}, \quad n \geq m
$$

for $m=2^{p+1}-1$ and

$$
H(n, m) \geq\left(\frac{\ln (m+1)}{2 \ln 2}+\frac{1}{6 \ln 2} \ln \frac{16}{9}\right) n-\bar{\beta}_{m}, \quad n \geq m
$$

for $m=3 \cdot 2^{p}-1$.

\section{Estimate of $H(m, m)$}

Following (3.1), define further

$$
\begin{gathered}
n_{21}=2 n_{11}+1, n_{22}=2 n_{11}+2, n_{23}=2 n_{12}+1, n_{24}=2 n_{12}+2, \\
m_{21}=2 m_{11}+1, m_{22}=2 m_{11}+2, m_{23}=2 m_{12}+1, m_{24}=2 m_{12}+2, \\
k_{21}=2 k_{11}+n_{11}, k_{22}=2 k_{11}+n_{11}+1, k_{23}=2 k_{12}+n_{12}, k_{24}=2 k_{12}+n_{12}+1 .
\end{gathered}
$$


Then by Theorem 2.1 and Corollary 2.1 again, there are polynomial Liénard systems of the form (2.1) which have $4^{2}$ properties $Z\left(n_{2 i}, m_{2 j}, k_{2 i}\right), i, j=1,2,3,4=2^{2}$, respectively, which give

$$
H\left(n_{2 i}, m_{2 j}\right) \geq k_{2 i}, i, j=1,2,3,4 \text {. }
$$

Also, we have obviously from (3.1) and (4.1)

$$
\begin{gathered}
k_{12}=k_{11}+1, \quad k_{24}=k_{23}+1, \quad k_{22}=k_{21}+1, \\
k_{21}=2^{2} k_{0}+2^{2} n_{0}+1, \quad k_{23}=2^{2} k_{0}+2^{2} n_{0}+4 .
\end{gathered}
$$

In general, we introduce three series $n_{p j}, m_{p j}$ and $k_{p j}$ for $p \geq 1$ and $j=1, \cdots, 2^{p}$. We do it by induction as follows:

$$
\begin{gathered}
n_{i+1,2 l-1}=2 n_{i l}+1, \quad n_{i+1,2 l}=2 n_{i l}+2, \\
m_{i+1,2 l-1}=2 m_{i l}+1, \quad m_{i+1,2 l}=2 m_{i l}+2, \\
k_{i+1,2 l-1}=2 k_{i l}+n_{i l}, \quad k_{i+1,2 l}=2 k_{i l}+n_{i l}+1, \\
l=1, \cdots, 2^{i}, \quad i \geq 1 .
\end{gathered}
$$

Then we can prove the following result.

Theorem 4.1. If there exists a polynomial system of the form (2.2) which has the property $Z\left(n_{0}, m_{0}, k_{0}\right)$, then for all $p \geq 1, i, j=1, \cdots, 2^{p}$ there are polynomial systems of the form (2.2) which have properties respectively $Z\left(n_{p i}, m_{p j}, k_{p i}\right)$ for all $p \geq 1, i, j=$ $1, \cdots, 2^{p}$, and therefore, $H\left(n_{p i}, m_{p j}\right) \geq k_{p i}$, where

$$
n_{p i}=2^{p}\left(n_{0}+1\right)-2+i, \quad m_{p j}=2^{p}\left(m_{0}+1\right)-2+j,
$$

and

$$
\begin{aligned}
k_{p 1}= & 2^{p}\left(k_{0}-1\right)+p 2^{p-1}\left(n_{0}+1\right)+1, \\
k_{p, 2^{p}}= & 2^{p}\left(k_{0}-1\right)+p 2^{p-1}\left(n_{0}+2\right)+1, \\
& k_{p 1}<k_{p 2}<\cdots<k_{p, 2^{p}} .
\end{aligned}
$$

Proof. The formulas for $n_{p i}$ and $m_{p j}$ in (4.3) follow from Lemma 3.1 in [16]. From (4.1) and (4.2) it is easy to prove by induction that $k_{p j} \geq k_{p i}$ for $1 \leq i<j \leq 2^{p}$. Hence, to finish the proof, it suffices to prove the two equalities in (4.4).

For the first equality, it is true for $p=1$. Suppose it is true for $p=i$. Then by (4.2) and the formula for $n_{i 1}$ we have

$$
k_{i+1,1}=2 k_{i, 1}+n_{i 1}=2 k_{i, 1}+2^{i}\left(n_{0}+1\right)-1
$$

which, together with the inductive assumption, yields that

$$
\begin{aligned}
k_{i+1,1} & =2\left(2^{i}\left(k_{0}-1\right)+i 2^{i-1}\left(n_{0}+1\right)+1\right)+2^{i}\left(n_{0}+1\right)-1 \\
& =2^{i+1}\left(k_{0}-1\right)+(i+1) 2^{i}\left(n_{0}+1\right)+1
\end{aligned}
$$


This shows that the equality is also true for $p=i+1$. Then the first equality in (4.4) follows.

The second one can be obtained in the same way since by (4.2) and (4.3)

$$
k_{i+1,2^{i+1}}=2 k_{i, 2^{i}}+n_{i, 2^{i}}+1=2 k_{i, 2^{i}}+2^{i}\left(n_{0}+2\right)-1 .
$$

This completes the proof.

In the following we take $n_{0}=m_{0}$ and suppose that there exists a polynomial system of the form (2.2) which has the property $Z\left(m_{0}, m_{0}, k_{0}\right)$. Then by Theorem 4.1 , we have

$$
H\left(m_{p i}, m_{p i}\right) \geq 2^{p}\left(k_{0}-1\right)+p\left(m_{0}+1\right) 2^{p-1}+1,
$$

where $m_{p i}=2^{p}\left(m_{0}+1\right)-2+i, p \geq 1,1 \leq i \leq 2^{p}$.

Let $m>m_{0}$ and $m \in S_{m_{0}}$. Then $m=m_{p i}$ for some $p \geq 1,1 \leq i \leq 2^{p}$. If $i=1$, then $m=2^{p}\left(m_{0}+1\right)-1$, or

$$
2^{p}=\frac{m+1}{m_{0}+1}, \quad p=\frac{1}{\ln 2} \ln \frac{m+1}{m_{0}+1} .
$$

Then by (4.5) we obtain

$$
H(m, m) \geq \frac{(m+1) \ln (m+1)}{2 \ln 2}+N\left(m_{0}, k_{0}\right)(m+1)+1,
$$

for $m=2^{p}\left(m_{0}+1\right)-1, p \geq 1$, where

$$
N\left(m_{0}, k_{0}\right)=\frac{k_{0}-1}{m_{0}+1}-\frac{\ln \left(m_{0}+1\right)}{2 \ln 2} .
$$

If $2 \leq i \leq 2^{p}$, then

$$
2^{p} \geq \frac{m+2}{m_{0}+2}, \quad p \geq \frac{1}{\ln 2} \ln \frac{m+2}{m_{0}+2} .
$$

By (4.5) again we obtain

$$
H(m, m) \geq N_{1}\left(m_{0}\right) \frac{(m+2) \ln (m+2)}{2 \ln 2}+N_{2}\left(m_{0}, k_{0}\right)(m+2)+1 \equiv H_{m_{0}}(m),
$$

where

$$
m \in S_{m_{0}}, \quad N_{1}\left(m_{0}\right)=\frac{m_{0}+1}{m_{0}+2}, \quad N_{2}\left(m_{0}, k_{0}\right)=\frac{k_{0}-1}{m_{0}+2}-\frac{N_{1}\left(m_{0}\right) \ln \left(m_{0}+2\right)}{2 \ln 2} .
$$

Similarly, by Theorem 4.1, we have

$$
H\left(m_{p, 2^{p}}, m_{p, 2^{p}}\right) \geq 2^{p}\left(k_{0}-1\right)+p\left(m_{0}+2\right) 2^{p-1}+1,
$$

where $m_{p, 2^{p}}=2^{p}\left(m_{0}+2\right)-2$, which gives

$$
H(m, m) \geq \frac{(m+2) \ln (m+2)}{2 \ln 2}+N\left(m_{0}+1, k_{0}\right)(m+2)+1,
$$


for $m=2^{p}\left(m_{0}+2\right)-2, \quad p \geq 1$.

By (4.6) and (4.8) we can obtain

Theorem 4.2. For any $k \geq 2$ there exist constants $B_{k}$ and $\bar{B}_{k}$ satisfying

$$
\lim _{k \rightarrow \infty} \frac{B_{k}}{\ln k}=\lim _{k \rightarrow \infty} \frac{\bar{B}_{k}}{\ln k}=-\frac{1}{2 \ln 2}
$$

such that

$$
H(m, m) \geq \frac{(m+1) \ln (m+1)}{2 \ln 2}+B_{k}(m+1)+1
$$

for $m=2^{p} k-1, p \geq 1$, and

$$
H(m, m) \geq \frac{(m+2) \ln (m+2)}{2 \ln 2}+\bar{B}_{k}(m+2)+1
$$

for $m=2^{p}(k+1)-2, p \geq 1$.

In particular, for $m=2^{p+1}-1, p \geq 1$, we have

$$
H(m, m) \geq \frac{(m+1) \ln (m+1)}{2 \ln 2}+1 .
$$

Proof. We need only to prove (4.9). In fact, by Lemma 2.1, we have the property $Z(3,3,5)$. Thus, taking $k_{0}=5, m_{0}=3$ in (4.6) we obtain (4.9) directly. The proof is ended.

By (4.7), we have further

Theorem 4.3. (1) For all $m \geq 3$ we have

$$
H(m, m) \geq \frac{(m+2) \ln (m+2)}{3 \ln 2}-\frac{m+2}{3}\left(1+\frac{\ln 3}{\ln 2}\right)+1 .
$$

$$
\lim _{m \rightarrow \infty} \inf \frac{H(m, m)}{(m+2) \ln (m+2)} \geq \frac{1}{2 \ln 2} .
$$

That is to say, $H(m, m)$ grows at least as rapidly as $\frac{1}{2 \ln 2}(m+2) \ln (m+2)$ as $m$ goes to infinity.

Proof. Using properties $Z(1,1,0)$ and $Z(2,2,1)$, by (4.7) we have $H(m, m) \geq H_{1}(m)$ for $m \in S_{1}, H(m, m) \geq H_{2}(m)$ for $m \in S_{2}$, where

$$
\begin{aligned}
H_{1}(m) & =\frac{(m+2) \ln (m+2)}{3 \ln 2}-\frac{m+2}{3}\left(1+\frac{\ln 3}{\ln 2}\right)+1 \\
& \leq \frac{3(m+2) \ln (m+2)}{8 \ln 2}-\frac{m+2}{3}\left(1+\frac{\ln 3}{\ln 2}\right)+1 \\
& \leq \frac{3(m+2) \ln (m+2)}{8 \ln 2}-\frac{3(m+2)}{4}+1=H_{2}(m) .
\end{aligned}
$$

It follows that for $m \in S_{1} \bigcup S_{2}$ we have $H(m, m) \geq H_{1}(m)$, which gives (4.10).

Now let $M$ be an arbitrary integer. Let

$$
\bar{N}_{1}(M)=\min \left\{N_{1}\left(m_{0}\right) \mid M \leq m_{0} \leq 2 M\right\}
$$




$$
\bar{N}_{2}(M)=\min \left\{N_{2}\left(m_{0}, 0\right) \mid M \leq m_{0} \leq 2 M\right\}
$$

Then by (4.7), we have $\bar{N}_{1}(M)=\frac{M+1}{M+2}$ and

$$
H(m, m) \geq \bar{N}_{1}(M) \frac{(m+2) \ln (m+2)}{2 \ln 2}+\bar{N}_{2}(M)(m+2)+1
$$

for all $m \in \bigcup_{m_{0}=M}^{2 M} S_{m_{0}}$. By $(\underline{3.3})$, the above inequality holds for all $m \geq M$. Therefore,

$$
\lim _{m \rightarrow \infty} \inf \frac{H(m, m)}{(m+2) \ln (m+2)} \geq \frac{\bar{N}_{1}(M)}{2 \ln 2} .
$$

Since $M$ is arbitrary (4.11) follows. This ends the proof.

\section{Estimate of $H(m \pm r, m)$}

Let $r$ be a positive integer. We can give an estimate of $H(m \pm r, m)$ using the result obtained in the previous section or the method used above. First, noting

$$
H(m+r, m) \geq H(m, m), \quad H(m-r, m) \geq H(m-r, m-r),
$$

we obtain by (4.9) and (4.11)

$$
H(m+r, m) \geq \frac{(m+1) \ln (m+1)}{2 \ln 2}+1
$$

for $m=2^{p+1}-1, p \geq 1$

$$
H(m-r, m) \geq \frac{(m+1-r) \ln (m+1-r)}{2 \ln 2}+1
$$

for $m=2^{p+1}-1+r, p \geq 1$; and

$$
\lim _{m \rightarrow \infty} \inf \frac{H(m \pm r, m)}{m \ln m} \geq \frac{1}{2 \ln 2} .
$$

However, for the case $H(m-r, m)$ using the above method, we can get more and better estimate. We describe the process briefly here.

First, suppose that there exists a polynomial system of the form (2.2) which has the property $Z\left(m_{0}-r, m_{0}, k_{0}\right)$. Then, as we do in Theorem 2.1, we can construct a system of the form (2.7) which has the property $Z\left(2 q, 2 m_{0}+1,2 k_{0}+q\right)$. It follows that $H\left(2 q, 2 m_{0}+1\right) \geq 2 k_{0}+q$. In order to obtain $H\left(2 m_{0}+1-r, 2 m_{0}+1\right) \geq 2 k_{0}+q$, we need to have $2 q \leq 2 m_{0}+1-r$ or $q \leq m_{0}-\left[\frac{r}{2}\right]$. We take $q=m_{0}-\left[\frac{r}{2}\right]$ and introduce

$$
m_{11}=2 m_{0}+1, m_{12}=2 m_{0}+2, k_{11}=2 k_{0}+m_{0}-\left[\frac{r}{2}\right]=k_{12} .
$$


Then we have properties $Z\left(m_{1 j}-r, m_{1 j}, k_{1 j}\right), j=1,2$. In general, define

$$
\begin{gathered}
m_{i+1,2 l-1}=2 m_{i l}+1, \quad m_{i+1,2 l}=2 m_{i l}+2, \\
k_{i+1,2 l-1}=k_{i+1,2 l}=2 k_{i l}+m_{i l}-\left[\frac{r}{2}\right], \\
l=1, \cdots, 2^{i}, \quad i \geq 1 .
\end{gathered}
$$

Then, as before, we have $H\left(m_{i j}-r, m_{i j}\right) \geq k_{i j}$ where

$$
\begin{gathered}
m_{i j}=2^{i}\left(m_{0}+1\right)-2+j, i \geq 1, j=1, \cdots, 2^{i}, \\
k_{i 1}=2^{i}\left(k_{0}-1-\left[\frac{r}{2}\right]\right)+i 2^{i-1}\left(m_{0}+1\right)+1+\left[\frac{r}{2}\right], \\
k_{i, 2^{i}}=2^{i}\left(k_{0}-2-\left[\frac{r}{2}\right]\right)+i 2^{i-1}\left(m_{0}+2\right)+2+\left[\frac{r}{2}\right], \\
k_{i 1} \leq k_{i 2} \leq \cdots \leq k_{i, 2^{i}} .
\end{gathered}
$$

Therefore, using (5.2), similar to (4.6), (4.8) and (4.7) we can obtain

$$
H(m-r, m) \geq \frac{(m+1) \ln (m+1)}{2 \ln 2}+N\left(m_{0}, k_{0}-\left[\frac{r}{2}\right]\right)(m+1)+1+\left[\frac{r}{2}\right]
$$

for $m=2^{i}\left(m_{0}+1\right)-1, i \geq 1$, and

$$
H(m-r, m) \geq \frac{(m+2) \ln (m+2)}{2 \ln 2}+N\left(m_{0}+1, k_{0}-1-\left[\frac{r}{2}\right]\right)(m+2)+2+\left[\frac{r}{2}\right]
$$

for $m=2^{i}\left(m_{0}+2\right)-2, i \geq 1$, and

$H(m-r, m) \geq N_{1}\left(m_{0}\right) \frac{(m+2) \ln (m+2)}{2 \ln 2}+N_{2}\left(m_{0}, k_{0}-\left[\frac{r}{2}\right]\right)(m+2)+1+\left[\frac{r}{2}\right] \equiv \bar{H}_{m_{0}}(m)$

for $m \in S_{m_{0}}$.

Using (5.3), (5.4) and (5.5), just similar to Theorems 4.2 and 4.3 we can obtain the following theorems.

Theorem 5.1. Let $r$ be a positive integer. For any $k \geq 2$ there exist constants $B_{k, r}$ and $\bar{B}_{k, r}$ satisfying

$$
\begin{gathered}
B_{k, r} \geq-\left[\frac{1}{k}\left(1+\left[\frac{r}{2}\right]\right)+\frac{\ln k}{2 \ln 2}\right], \\
\bar{B}_{k, r} \geq-\left[\frac{1}{k+1}\left(2+\left[\frac{r}{2}\right]\right)+\frac{\ln (k+1)}{2 \ln 2}\right],
\end{gathered}
$$

such that

$$
H(m-r, m) \geq \frac{(m+1) \ln (m+1)}{2 \ln 2}+B_{k, r}(m+1)+1+\left[\frac{r}{2}\right]
$$

for $m=2^{p} k-1, p \geq 1$, and

$$
H(m-r, m) \geq \frac{(m+2) \ln (m+2)}{2 \ln 2}+\bar{B}_{k, r}(m+2)+2+\left[\frac{r}{2}\right]
$$

for $m=2^{p}(k+1)-2, p \geq 1$.

Theorem 5.2. (1) For $m=2^{p+1}-1, p \geq 1$, we have

$$
H(m-1, m) \geq \frac{(m+1) \ln (m+1)}{2 \ln 2}+1 .
$$


(2) For all $m \geq 3$ we have

$$
H(m-1, m) \geq \frac{(m+2) \ln (m+2)}{3 \ln 2}-\frac{m+2}{3}\left(1+\frac{\ln 3}{\ln 2}\right)+1 .
$$

We mention that in Theorem 5.2 we have taken $r=1$. In this case, $H(m-1, m)$ denotes the maximal number of limit cycles of polynomial Liénard systems of degree $m$.

\section{References}

[1] A. Liénard, Etude des Oscillations Entretenues, Rev. gen. electr., 23(1928) 901 - 912.

[2] N. Glade, L. Forest and J. Demongeot, Liénard systems and potential-Hamiltonian decomposition III - applications, Comptes Rendus Mathematique. 344(2007) 253-258.

[3] J. Llibre. A survey on the limit cycles of the generalization polynomial Linard differential equations. Mathematical models in engineering, biology and medicine, 224-233, AIP Conf. Proc., 1124, Amer. Inst. Phys., Melville, NY, 2009.

[4] J. Llibre, A.C. Mereu, M.A. Teixeira, Limit cycles of the generalized polynomial Liénard differential equations, Math. Proc. Cambridge Philos. Soc. 148(2010) 363383.

[5] T. R. Blows, N. G. Lloyd, The number of small-amplitude limit cycles of Liénard equations, Math. Proc. Camb. Phil. Soc. 95(1984) 359-366.

[6] M. Han, Y. Tian, and P. Yu, Small-amplitude limit cycles of polynomial Liénard systems, Preprint, 2011.

[7] M. Han, Liapunov constants and Hopf cyclicity of Lineard systems, Ann. of Diff. Eqs. 15(1999) 113-126.

[8] F. Dumortier, C. Li, Perturbations from an elliptic Hamiltonian of degree four: (IV) Figure eight loop, J. Diff. Eqns. 188 (2003) 512-554.

[9] C. J. Christopher, S. Lynch, Small-amplitude limit cycle bifurcations for Liénard systems with quadratic or cubic damping or restoring forces, Nonlinearity 12(1999) 1099-1112.

[10] J. Yang, M. Han, V.G. Romanovski, Limit cycle bifurcations of some Liénard systems, J. Math. Anal. Appl. 366(2010) 242-255. 
[11] M. Han, H. Zang, J. Yang, Limit cycle bifurcations by perturbing a cuspidal loop in a Hamiltonian system, J. Diff. Equat. 246(2009) 129-163.

[12] J. Yang, M. Han, Limit cycle bifurcations of some Liénard systems with a nilpotent cusp, Int. J. Bifur. Chaos 11(2010) 3829-3839.

[13] M. Han, H. Yan, J. Yang, C. Lhotka, On the Number of Limit Cycles of Some Liénard Systems, Can. Appl. Math. Q. 17(2009) 61-83.

[14] P. Yu, M. Han, Limit cycles in generalized Liénard systems. Chaos, Solitons \& Fractals 30(2006) 1048-1068.

[15] J. Yang, M. Han, Limit cycle bifurcations of some Liénard systems with a cuspidal loop and a homoclinic loop, Chao, Soliton \& Fractal 44(2011) 269-289.

[16] M. Han, J. Li, Lower bounds for the Hilbert number of polynomial systems, Submitted

[17] J. Li. Hilbert's 16th problem and bifurcations of planar polynomial vector fields. Internat. J. Bifur. Chaos Appl. Sci. Engrg. 13(2003) 47-106.

[18] L. Gavrilov, Petrov modules and zeros of Abelian integrals, Bull. des Sciences Math. $122(1998) 571-584$.

[19] J. Llibre, X. Zhang, On the number of limit cycles for some perturbed Hamiltonian polynomial systems, Dyn. Contin. Discr. Impuls. Syst. Series A: Math. Anal. 8(2001) 161-181.

[20] C. Li, W. Li, J. Llibre, Z. Zhang, Polynomial systems: A lower bound for the weakened 16th Hilbert problem, Extracta Math. 16(2001) 441-447.

[21] Ye, Y. et al., Theory of Limit Cycles, Transl. Math. Monogr., vol.66, Amer. Math. Soc., Providence, RI., 1986. 